\title{
REPERCUSIONES PSICOPATOLÓGICAS DE LA VIOLENCIA DOMÉSTICA EN LA MUJER: UN ESTUDIO DESCRIPTIVO
}

\author{
Enrique Echeburúa; Paz de Corral, \\ Pedro Javier Amor; Belén Sarasua \\ - Irene Zubizarreta \\ Universidad del Pais Vasco
}

\begin{abstract}
RESUMEN
El principal objetivo de este trabajo ha sido estudiar diferentes variables sociodemográficas y psicopatológicas en una muestra de 164 víctimas de maltrato doméstico en tratamiento. Asimismo se hace una comparación en todas las variables estudiadas entre las victimas de maltrato físico $(N=93)$ y las de maltrato psicológico $(N=71)$. Los resultados ponen de manifiesto la existencia de repercusiones psicopatológicas importantes en el ámbito de la ansiedad -el trastorno de estrés postraumático especialmentey de la depresión y en el funcionamiento en la vida diaria, sin que haya diferencias significativas entre ambos grupos. Se senalan nuevas líneas de investigación en este ámbito.
\end{abstract}

Palabras-clave: Mujeres maltratadas. Trastorno de estrés postraumático. Maltrato físico. Maltrato psicológico.

\section{SUMMARY}

The aim of this paper has been to assess the sociodemographic and psychopathological variables in a sample of 164 battered women who were under psychological treatment. Scores of both physical $(N=93)$ and psychological $(N=71)$ battered women were compared. The results showed a high level of anxiety-posttraumatic stress disorder- and depression symptoms, as well as a general maladaptative level to daily life activities. Differences between the two groups were not observed. Some suggestions in order to further research are pointed out.

Key words: Battered women. Posttraumatic stress disorder. Physical battered women. Psychological battered women

Correspondencia: Prof. Enrique Echeburúa. Departamento de Personalidad, Evaluación y Tratamientos Psicológicos. Facultad de Psicologla. Universidad del País Vasco. Avda. de Tolosa, 70. 20009 San Sebastián. 


\section{INTRODUCCIÓN}

El maltrato doméstico a las mujeres se ha convertido en un problema social debido a su gran incidencia en la población y a la gravedad de las secuelas tanto fisicas como psicológicas producidas en las victimas. La violencia en el hogar supone la causa más común de lesiones en la mujer, más incluso que en el caso de los accidentes de coche, de los robos y de las violaciones (Stark y Flitcraft, 1988). Al contrario de lo que sucede con los hombres, más de las dos terceras partes de los actos violentos perpetrados contra las mujeres son cometidos por personas próximas a ellas (Rojas Marcos, 1995).

No es casual que la violencia surja frecuentemente en el hogar. Las agresiones repetidas y prolongadas se producen sobre todo en situaciones de cautiverio, cuando la víctima es incapaz de escapar del control del agresor al estar sujeta a él por la fuerza física o por vínculos económicos, legales, sociales o emocionales (Rojas Marcos, 1995).

Hoy en dia es difícil estimar la verdadera incidencia del maltrato doméstico. Los datos existentes son poco fiables y sólo se denuncia una minima parte de los casos (entre un 10\% y un $30 \%$ de los sucesos reales). Hasta hace unos pocos años la violencia en el hogar se consideraba como un asunto privado que pertenecia a la intimidad familiar y no debía aflorar al exterior (Villavicencio, 1993). Según los estudios más rigurosos llevados a cabo en EE UU (O'Leary y Arias, 1988; Stith, Williams y Rosen, 1992; Straus y Gelles, 1990), la tasa de prevalencia del maltrato doméstico oscila entre el $15 \%$ y el $30 \%$ de la población femenina.

El maltrato doméstico se refiere a las agresiones físicas, psíquicas, sexuales o de otra indole, llevadas a cabo reiteradamente por parte de un familiar (habitualmente el marido), y que causan daño físico y/o psiquico y vulneran la libertad de otra persona (habitualmente la esposa) (Echeburúa, Corral, Sarasua, Zubizarreta y Sauca, 1990).

Una de las características singulares del maltrato doméstico es la cronicidad. Por término medio las mujeres permanecen en la situación de violencia durante un periodo no inferior a 10 años (Zubizarreta, Sarasua, Echeburúa, Corral, Sauca y Emparanza, 1994). La búsqueda tardia de ayuda terapéutica se explica por razones económicas (dependencia del marido), sociales (la opinión de los demás), familiares (la protección de los hijos) y psicológicas (la minimización del problema, el miedo, la indefensión generada, la resistencia a reconocer el fracaso de la relación, el temor al futuro en soledad, etc.) (Saunders, 1994; Wodarski, 1987).

La victimización -el hecho de ser víctima de un delito- puede causar unas repercusiones psicológicas muy negativas en la estabilidad emocional de las personas afectadas. En concreto, el desarrollo del estrés postraumático como consecuencia de cualquier delito lo experimenta el $25 \%$ de todas las victimas, pero este porcentaje puede ascender hasta el $50-60 \%$ en el caso de las mujeres agredidas sexualmente (Corral, Echeburúa, Sarasua y Zubizarreta, 1992) y hasta el $51 \%$ en el caso de las víctimas de maltrato doméstico (Zubizarreta et al., 1994).

Los cuadros clínicos más frecuentemente relacionados con los efectos de la violencia familiar son la depresión (Campbell, Sullivan y Davidson, 1995; Rosewater, 1988; Sato y Heiby, 1992; Straus, 1987) y el trastorno de estrés postraumático (Douglas, 1987; Dutton, 1992; Dutton-Douglas, Burghardt, Perrin y Chrestman, 1994; Houskamp y Foy, 1991; Kusher, Riggs, Foa y Miller, 1992; Walker, 1989; Zubizarreta et al., 1994).

También pueden aparecer sintomas de elevada ansiedad, baja autoestima e indaptación en diferentes áreas de la vida cotidiana: trabajo, vida social, relación con los hijos, etc. Además, y de forma simultánea, pueden surgir problemas temporales o permanentes de abuso de alcohol y fármacos, a modo de estrategia de afrontamiento inadecuada para hacer frente a los problemas planteados.

El objetivo principal de esta investigación es describir, desde un punto de vista sociodemográfico y psicopatológico, las caracteristicas de una muestra amplia de mujeres maltratadas que acuden a los centros de atención psicológica en busca de ayuda terapéutica. Se trata también de delimitar las diferencias existentes entre las victimas de violencia física y las víctimas de maltrato psicológico. Por último, se estudian las correlaciones existentes entre las variables sociodemográficas y las psicopatológicas entre sí. 
Este estudio forma parte de una investigación más amplia sobre el tratamiento de la violencia familiar, referido tanto a victimas (Echeburúa, Corral, Sarasua y Zubizarreta, 1996) como a maltratadores en el hogar (Echeburúa y Fernández-Montalvo, 1997).

\section{MÉTODO}

\section{Sujetos}

La muestra de sujetos de este estudio consta de 164 mujeres victimas de maltrato doméstico que han acudido en busca de tratamiento a los Centros de Asistencia Psicológica para Víctimas de Violencia Familiar del Pais Vasco (Bilbao y Vitoria) durante el período de tiempo comprendido entre mayo de 1994 y diciembre de 1996.

Los criterios de admisión para este estudio han sido que la paciente sea una mujer adulta, que acuda voluntariamente a la terapia y que sea víctima de algún tipo de maltrato, ya sea psicológico o físico, en la relación de pareja. A efectos de homogeneizar la muestra, el único criterio de exclusión ha sido la presencia de una adicción, trastorno mental o enfermedad orgánica grave.

\section{Diseño}

En el estudio piloto llevado a cabo sobre la eficacia del tratamiento cognitivo-conductual del trastorno de estrés postraumático en víctimas de maltrato doméstico (Echeburúa et al., 1996), se ha utilizado un diseño experimental de medidas repetidas (pretratamiento, postratamiento y seguimientos de $1,3,6$ y 12 meses), con un único grupo experimental y con un número de sujetos $(N=62)$ menor que en este estudio, debido a la existencia de unos criterios de selección más restrictivos.

Dado el carácter descriptivo de esta investigación, y a efectos de dar representatividad a los datos analizados y de aumentar la potencialidad de los análisis estadisticos, se han incluido todos los sujetos que han podido ser evaluados, independientemente de que hayan formado parte o no del estudio piloto sobre tratamiento.

\section{Medidas de evaluación}

Los instrumentos de evaluación empleados en esta investigación han sido entrevistas estructuradas y medidas de autoinforme.

\section{a) Entrevistas}

La Entrevista Semiestructurada sobre Maltrato Doméstico (Echeburúa, Corral, Sarasua, Zubizarreta y Sauca, 1994, no publicada) permite evaluar las características demográficas de la victima, la historia de victimización y de trastornos psicopatológicos y las circunstancias del maltrato doméstico, asi como la expresión emocional de la víctima y la reacción del entorno familiar y social ante los acontecimientos vividos.

La Escala de Gravedad de Sintomas del Trastomo de Estrés Postraumático (Echeburúa, Corral, Sarasua, Zubizarreta y Sauca, 1994) es una entrevista estructurada que sirve para evaluar los sintomas y la intensidad del trastorno de estrés postraumático según los criterios diagnósticos del DSM-IV (American Psychiatric Association, 1994).

Esta escala, estructurada en un formato de tipo Likert de 0 a 3 según la frecuencia $e$ intensidad de los sintomas, consta de 17 items, de los que 5 hacen referencia a los sintomas de reexperimentación, 7 a los de evitación y 5 a los de hiperactivación. El rango es de 0 a 51 en la escala global; de 0 a 15 en la subescala de reexperimentación, de 0 a 21 en la de evitación y de 0 a 15 en la de activación.

La existencia del diagnóstico del trastorno de estrés postraumático, según los requisitos del DSM-IV (APA, 1994), exige una puntuación global mínima de 12, con una distribución de 2 puntos en la escala de reexperimentación (se requiere un sintoma), de 6 en la de evitación (se necesitan tres sintomas) y de 4 en la de activación (se requieren dos sintomas).

\section{b) Evaluación de otros sintomas psico- patológicos asociados}

Además de las medidas del trastorno de estrés postraumático, se han evaluado otros indicadores psicopatógicos asociados habitualmente al maltrato doméstico: la ansiedad, la depresión, la autoestima y el grado de inadaptación a la vida cotidiana.

El Inventario de Ansiedad Estado-Rasgo (STAI) (Spielberger, Gorsuch y Lushene, 1970; 
versión española de TEA, 1988) consta de 20 items relacionados con la ansiedad-rasgo y de otros 20 relacionados con la ansiedad-estado. El rango de las puntuaciones es de 0 a 60 en cada escala. La fiabilidad test-retest es de 0,81 en la ansiedad-rasgo y, como es lógico, bastante más baja en la ansiedad-estado $(r=0,40)$. El coeficiente alfa de consistencia interna oscila de 0,83 a 0,92 .

En esta investigación se ha empleado sólo la escala de ansiedad-estado, en donde el rango de la escala es de 0 a 60 y la puntuación media de la población es de 23,30 $(D T=11,93)$.

El Inventario de Depresión de Beck (BDI) (Beck, Rush, Shaw y Emery, 1979; versión española de Vázquez y Sanz, 1991) es un instrumento autoaplicado que consta de 21 items y mide la intensidad de los síntomas depresivos. El rango de las puntuaciones es de 0 a 63. El punto de corte más utilizado para discriminar a la población sana de la población aquejada de sintomatologia depresiva es 18.

El BDI, a diferencia de la escala de Hamilton (1960), se centra en los síntomas más cognitivos de la depresión (2/3 de los ítems), pero mucho menos en los aspectos somáticos o conductuales (1/3 de los items).

El coeficiente de fiabilidad por el método de las dos mitades es de 0,93. Desde la perspectiva de la validez convergente, la correlación con la evaluación clínica de la depresión oscila de 0,62 a 0,66. La versión utilizada en este estudio puede encontrarse en Echeburúa (1995).

La Escala de Depresión de Hamilton (HRS) (Hamilton, 1960) (versión heteroaplicada de 21 items). El punto de corte es de 18 (rango: 0-52). En esta escala, a diferencia del $B D I$, se da mayor importancia a los síntomas somáticos y comportamentales de la depresión que a las manifestaciones cognitivas.

Este instrumento posee una buena fiabilidad entre observadores $(r=0,90)$, tiene un buen nivel de validez concurrente con el $B D I(r=0,60)$ y es muy sensible -quizá el que más- a los cambios terapéuticos.

La Escala de Autoestima (Rosenberg, 1965) tiene por objetivo evaluar el sentimiento de satisfacción que una persona tiene consigo misma. Este instrumento consta de 10 items estructurados en un formato de tipo Likert que oscila de 1 a 4 en relación con el grado de acuerdo. La mitad de los items están planteados de forma afirmativa y la otra mitad de forma negativa, con el objetivo de controlar la aquiescencia. La puntuación total varia entre 10 y 40 , con una puntuación tanto mayor cuanto mayor es la autoestima. El punto de corte en la población adulta es de 29 .

La fiabilidad test-restest es de 0,85 y el coeficiente alfa de consistencia interna es de 0,92 . La validez convergente y la validez discriminante son asimismo satisfactorias (cfr. Silber y Tippet, 1965; Zubizarreta et al., 1994). La versión española utilizada en este estudio puede encontrarse en Echeburúa (1995).

La Escala de Inadaptación (Echeburúa y Corral, 1988) valora el grado en que la situación de maltrato afecta a diferentes áreas de la vida cotidiana: trabajo, vida social, tiempo libre, relación de pareja y vida familiar. Este instrumento, con 6 ítems que oscilan de 1 a 6 en una escala de tipo Likert, consta asimismo de una subescala global que refleja el grado de inadaptación global a la vida cotidiana. El rango total de la escala es de 6 a 36, con una puntuación tanto mayor cuanto mayor es la inadaptación. La escala utilizada en este estudio puede encontrarse en Echeburúa (1995).

\section{Procedimiento}

En este estudio se han utilizado estadisticos descriptivos para determinar la frecuencia de los síntomas que se producen como consecuencia de la violencia familiar. Para un estudio más detallado la muestra global se ha dividido en dos submuestras en función del tipo de violencia experimentado por las victimas: maltrato físico y maltrato psicológico.

En primer lugar, se analizan las medias y las desviaciones típicas en relación con las variables sociodemográficas y las circunstancias del maltrato doméstico. En segundo lugar, desde una perspectiva categorial, se estudian los porcentajes de víctimas afectadas por el trastorno de estrés postraumático, tanto en la muestra total como en las dos submuestras (maltrato fisico y maltrato psicológico). En tercer lugar, desde una perspectiva dimensional, se analizan las medias y las desviaciones típicas del nivel de gravedad total del trastorno de estrés postraumático y del 
REPERCUSIONES DE LA VIOLENCIA DOMÉSTICA EN MUJERES

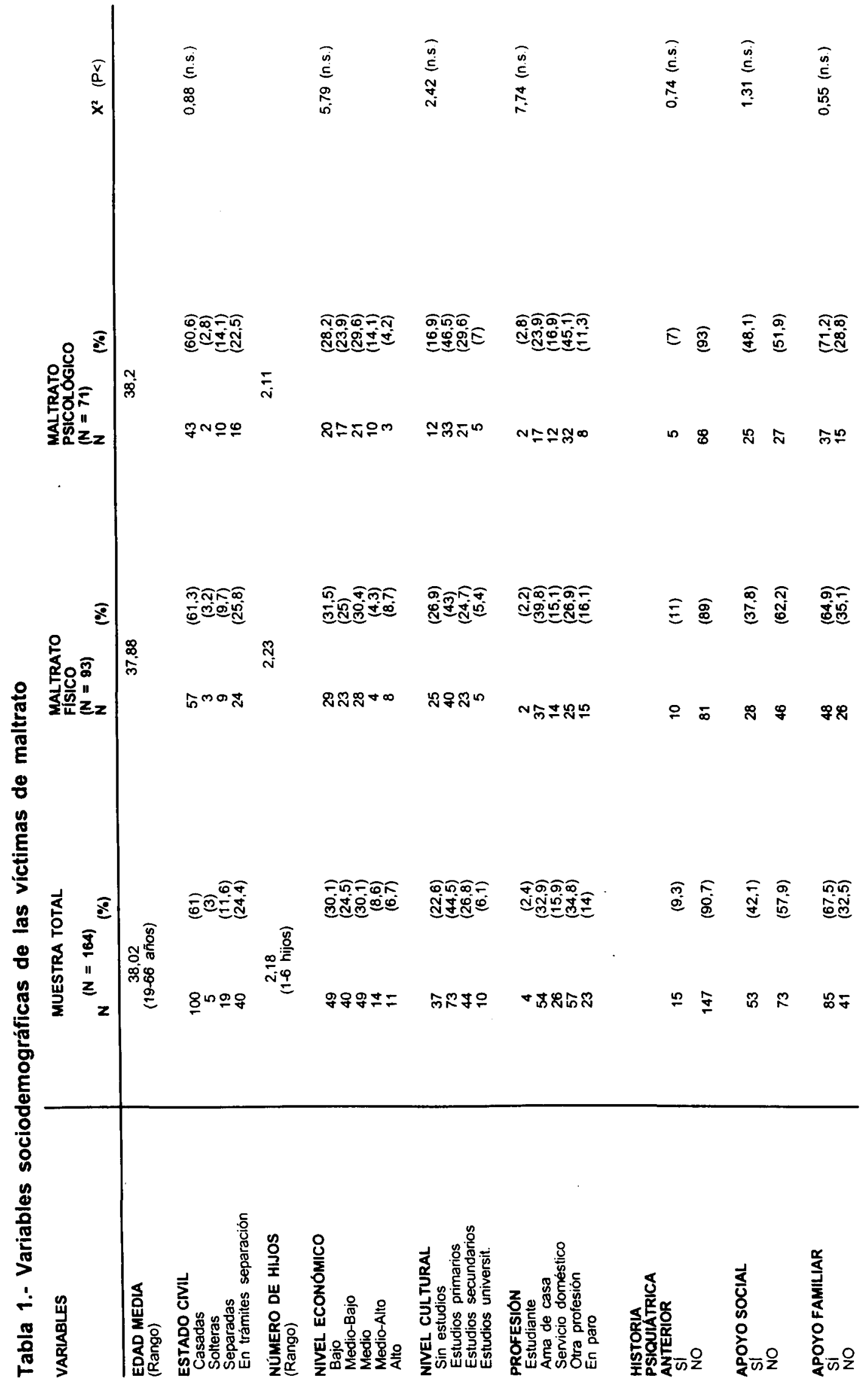


nivel de intensidad de los tres grupos de sintomas (reexperimentación, evitación y activación). $Y$ por último, se muestran las medias y desviaciones tipicas obtenidas en cada una de las variables psicopatológicas evaluadas (ansiedad, depresión y autoestima), con un anáisis comparativo, al igual que en las variables anteriores, de las dos submuestras estudiadas.

El número de sujetos utilizado ha sido de 164. Sin embargo, en el estudio de las variables psicopatológicas la muestra es algo menor $(N=126)$. La reducción de sujetos en este caso es debida a que algunas personas $(N=38)$ que acudieron a los Centros, en donde se les abrió una historia clinica en relación con las variables sociodemográficas y las circunstancias del maltrato, no continuaron posteriormente con las sesiones de evaluación y tratamiento.

\section{RESULTADOS}

En los párrafos siguientes se van a presentar los resultados referidos, en primer lugar, a las variables sociodemográficas y de maltrato. En segundo lugar, se van a exponer los datos correspondientes a la presencia del trastorno de estrés postraumático y de las variables que lo modulan. Por último, se van a señalar los resultados en relación con las variables psicopatológicas y de adaptación de las víctimas estudiadas.

\section{Variables sociodemográficas}

Las características sociodemográficas de la muestra total $(N=164)$ aparecen descritas en la tabla 1, con una referencia específica a los dos tipos de violencia, que aparecen relativamente equilibrados (maltrato físico, $57 \%$; maltrato psicológico, $43 \%$ ) en el conjunto de las victimas.

Como se puede observar, la edad media de la muestra total es de 38 años (con un rango que oscila entre los 19 y los 66 años) y eł número medio de hijos es de 2 . En cuanto al estado civil, la mayoria de las víctimas permanecen casadas, pero hay un grupo significativo de ellas que están separadas $(11 \%)$ o en trámites de separación (24\%).

El nivel de instrucción de la mayoría es más bien bajo, con un predominio claro de victimas sin estudios o con estudios primarios $(67 \%)$ y con sólo un $6 \%$ de mujeres con formación universitaria. Asimismo el nivel socioeconómico de los casos estudiados oscila entre la clase baja y la clase media. En cuanto a la ocupación laboral, predominan las amas de casa y las empleadas domésticas, si bien hay hasta un $35 \%$ de víctimas que ejercen profesiones diversas y heterogéneas.

Las víctimas suelen contar con algún tipo de redes de apoyo, que son más intensas en el ámbito familiar (67\%) que en el social (42\%).

Sólo una pequeña parte de las víctimas (un $9 \%)$ ha tenido una historia psiquiátrica anterior, que se especifica en la tabla 2, referida sobre todo al ámbito de las alteraciones emocionales (ansiedad y depresión). Se trata, por tanto, en general de mujeres equilibradas mentalmente que sufren de trastornos psicológicos actualmente como consecuencia de una situación de maltrato crónico.

Tabla 2.- Historia de asistencia psiquiátrica en mujeres maltratadas

\begin{tabular}{l|cc}
$\begin{array}{l}\text { TRASTORNO DE } \\
\text { CONDUCTA }\end{array}$ & N & $(\%)$ \\
\hline * DEPRESION & 6 & $(40)$ \\
* ANSIEDAD & 3 & $(20)$ \\
* AGRESION SEXUAL & 2 & $(13,6)$ \\
* INCESTO & 1 & $(6,6)$ \\
* CONSUMO ABUSIVO & & \\
DE ALCOHOL & 1 & $(6,6)$ \\
* FOBIA & 1 & $(6,6)$ \\
SIN PRECISAR & 1 & $(6,6)$ \\
T O T A L & 15 & $(100)$
\end{tabular}

Al comparar a las víctimas de maltrato físico y psicológico, en ninguna de las variables sociodemográficas evaluadas se han encontrado diferencias significativas entre ellas.

\section{Variables de maltrato}

En cuanto a la duración de la situación de violencia, un primer dato es que la situación de maltrato es más bien crónica y de larga evolución. Al margen de que el rango es muy 
REPERCUSIONES DE LA VIOLENCIA DOMESTICA EN MUJERES

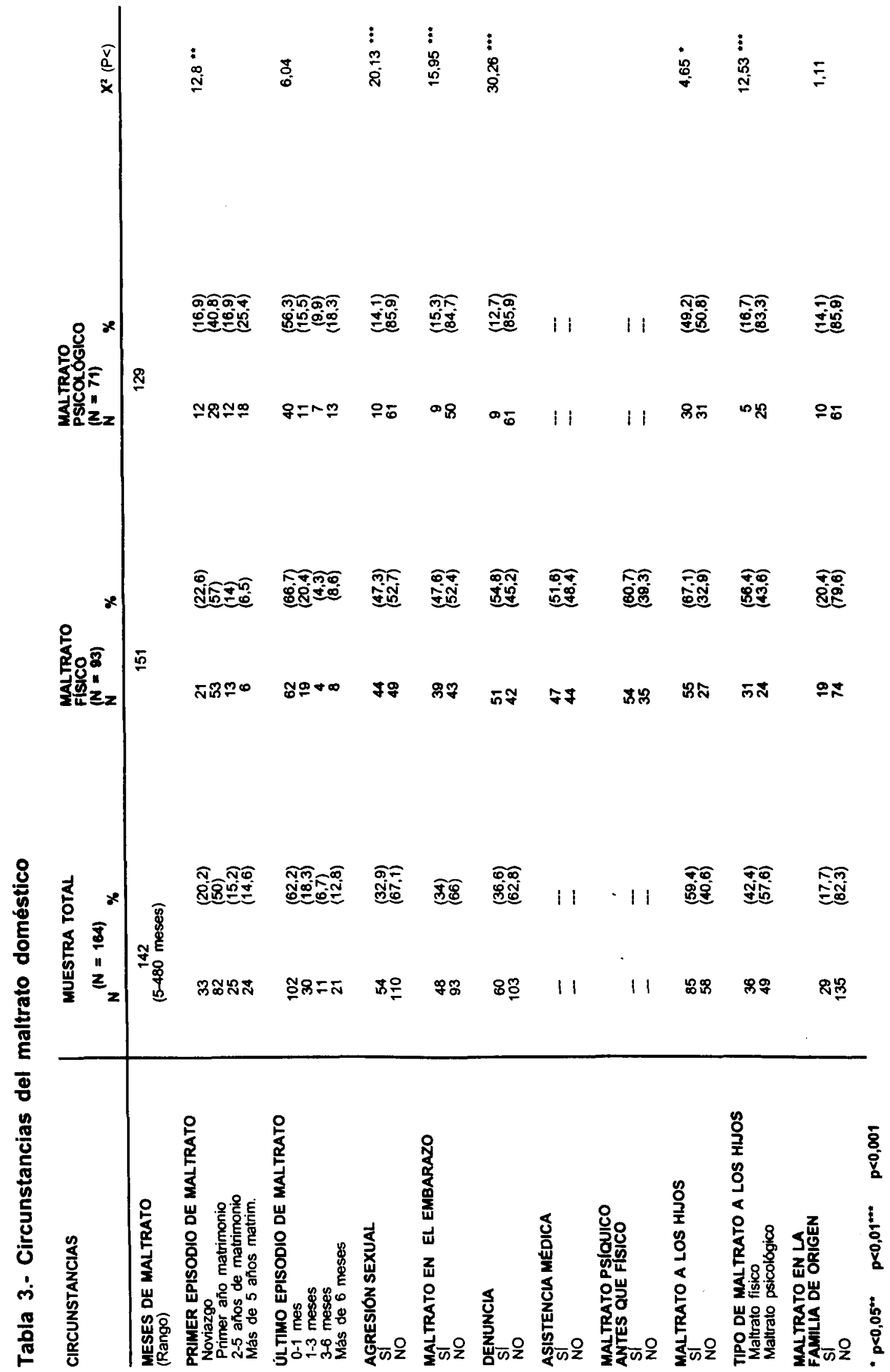


amplio, la duración media del maltrato es de 12 años. Un segundo dato es que el maltrato es de instauración precoz en la relación de pareja. En concreto, en el $70 \%$ de los casos el maltrato comienza en el primer año de matrimonio e incluso en el noviazgo. En general, en la submuestra de maltrato fisico la antigüedad del problema suele ser mayor que en la de maltrato psicológico $\left(X^{2}=12,8 ; p<0,01\right)$. En casi todos los casos, independientemente del subtipo de maltrato, los últimos episodios violentos han sido muy recientes (tabla 3 ).

La intensidad de la violencia familiar queda reflejada por el hecho de que la mitad de las víctimas de maltrato físico han requerido asistencia médica y de que la tercera parte de todas las pacientes, si bien con más frecuencia en el caso del maltrato físico, han experimentado relaciones sexuales forzadas y/o han sido víctimas de maltrato incluso durante los embarazos.

La violencia contra la mujer tiende a hacerse extensiva a los hijos. En concreto, el maltrato infantil por parte del hombre se da en más de la mitad de la muestra, pero es mayor en el caso de la submuestra de maltrato físico $(67 \%)$ que en el caso de la de maltrato psicológico (49\%) $\left(X^{2}=4,65 ; p<0,05\right)$. Y la modalidad de maltrato ejercida contra los hijos suele coincidir con el tipo de maltrato sufrido por la mujer.

Tabla 4.- Trastorno de estrés postraumático en víctimas de maltrato doméstico

\begin{tabular}{|c|c|c|c|}
\hline $\begin{array}{l}\text { TRASTORNO } \\
\text { DE ESTRES } \\
\text { POSTRAU- } \\
\text { MATICO }\end{array}$ & $\begin{array}{l}\text { MUESTRA } \\
\text { TOTAL } \\
(N=126) \\
N(\%)\end{array}$ & $\begin{array}{l}\text { MALTRATO } \\
\text { FISICO } \\
(N=74) \\
N(\%)\end{array}$ & $\begin{array}{l}\text { MALTRATO } \\
\text { PSICOLO- } \\
\text { GICO } \\
(N=52) \\
N \quad(\%)\end{array}$ \\
\hline si & $69(54,8)$ & $40(31,7)$ & $29(23)$ \\
\hline NO & $57(45,2)$ & $34(27)$ & $23(18,3)$ \\
\hline
\end{tabular}

Desde la perspectiva de los antecedentes familiares, sólo una pequeña parte de la muestra -el $18 \%$ - ha sufrido o ha sido testigo de maltrato en la familia de origen. Por ello, la violencia familiar no se articula necesariamente en una cadena de transmisión.
En cuanto a las denuncias, que se dan en una tercera parte del total de las victimas, se presentan con mucha más frecuencia en el caso del maltrato físico $(55 \%)$ que en el del maltrato psicológico $(13 \%)\left(X^{2}=30,26 ; p<0,001\right)$. Este último tiende a percibirse por parte de las víctimas como una conducta privada de la pareja que no debe aflorar al exterior y que, además, resulta dificil de demostrar.

\section{Trastorno de estrés postraumático}

Las victimas aquejadas del trastorno de estrés postraumático, según los criterios diagnósticos del DSM-IV (APA, 1994), suponen el $55 \%$ de la muestra total de las victimas de maltrato, sin que existan diferencias significativas, desde una perspectiva categorial, entre las dos submuestras (tabla 4). No es menos grave, por ello, bajo este punto de vista, ser víctima de maltrato psicológico que de maltrato fisico.

Desde una perspectiva dimensional, en la tabla 5 figuran las puntuaciones obtenidas por las victimas de maltrato en la Escala de Gravedad de Sintomas del Trastorno de Estrés Postraumático, tanto a nivel global como a nivel de los principales grupos de sintomas.

Como puede observarse, la intensidad global del trastorno de estrés postraumático en las víctimas de violencia familiar es más bien las victimas de maltrato en la Escala de Gravedad de Sintomas del Trastorno de Esalta. Una vez más, no hay diferencias significativas entre las dos submuestras ni a nivel global ni a nivel de los diferentes grupos de sintomas.

Por otra parte, el trastorno de estrés postraumático está asociado a una mayor inadaptación global $(r=0,48 ; p<0,001)$, a la existencia de relaciones sexuales forzadas $(r=0,26 ; p<0,01)$, a la presencia de maltrato incluso durante los embarazos $(r=0,16 ; p<0,05)$ y a la carencia de apoyo social y familiar $(r=-0,14 ; p<0,10)$.

\section{Variables psicopatológicas y de adaptación}

Los principales resultados obtenidos en las variables psicopatológicas y de inadaptación estudiadas aparecen descritos en la tabla 6. En general, las victimas de maltrato presentan un nivel muy elevado de sintomas psicopatológicos en el momento de la búsqueda de ayu- 
Tabla 5.- Medias (y desviaciones típicas) obtenidas en la escala de gravedad de sintomas del trastorno de estrés postraumático

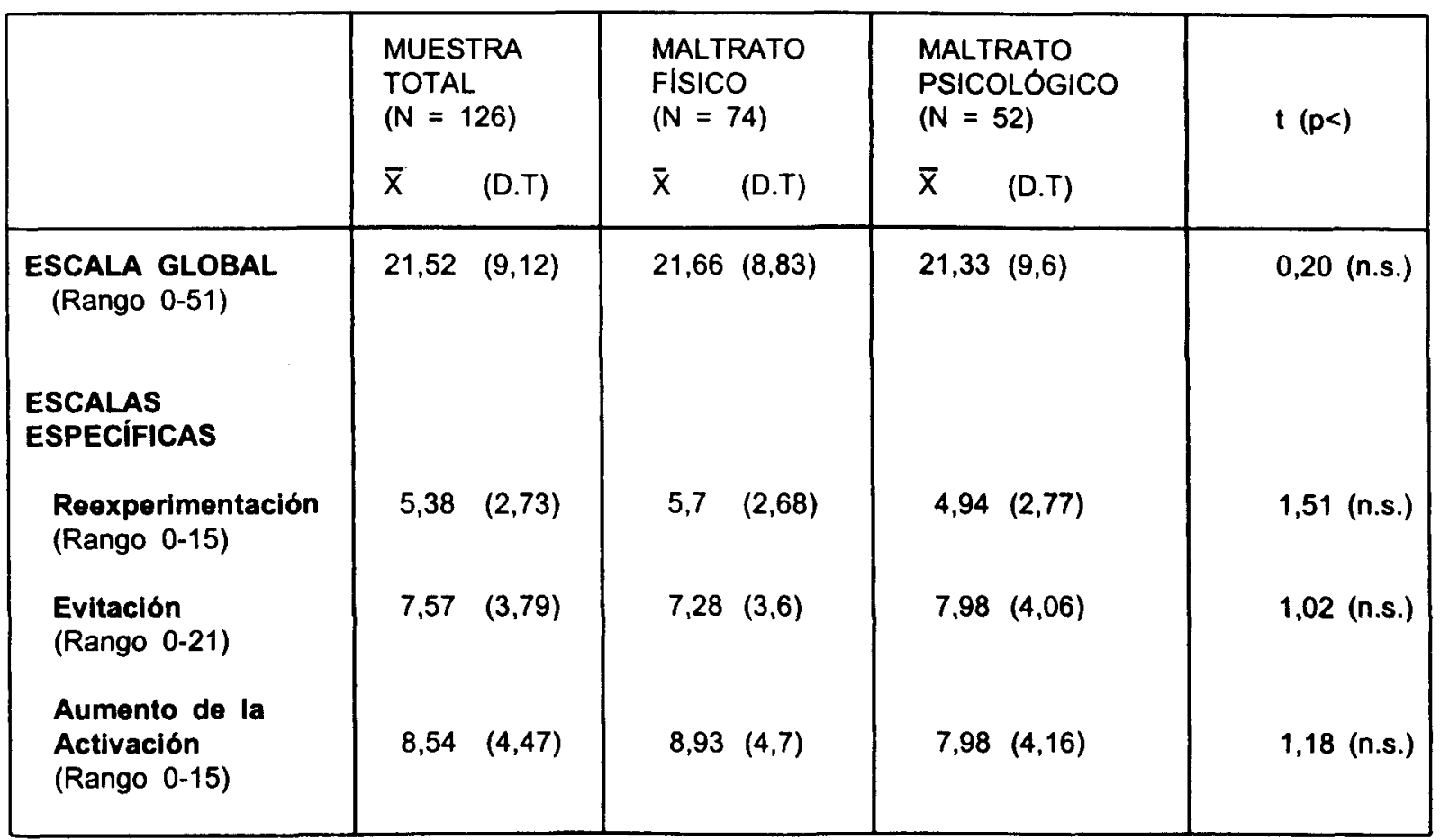

Tabla 6.- Puntuaciones en las variables psicopatológicas en las mujeres víctimas de maltrato doméstico

\begin{tabular}{|c|c|c|c|c|}
\hline & $\begin{array}{l}\text { MUESTRA } \\
\text { TOTAL } \\
(\mathrm{N}=126) \\
\bar{X} \quad \text { (D.T) }\end{array}$ & $\begin{array}{l}\text { MALTRATO } \\
\text { FISICO } \\
(N=74) \\
\bar{X} \quad \text { (D.T) }\end{array}$ & $\begin{array}{l}\text { MALTRATO } \\
\text { PSICOLÓGICO } \\
(N=52) \\
\bar{X} \quad \text { (D.T) }\end{array}$ & $t(p<)$ \\
\hline $\begin{array}{l}\text { ANSIEDAD-ESTADO } \\
\text { (STAI-E) } \\
\text { (Rango 0-60) }\end{array}$ & $42,79(11,67)$ & $43,3 \quad(11,6)$ & $42,07(11,79)$ & 0,58 (n.s.) \\
\hline $\begin{array}{l}\text { INVENTARIO DE } \\
\text { DEPRESION (BDI) } \\
\text { (Rango 0-63) }\end{array}$ & $20,76(9,89)$ & $20(9,96)$ & $21,79(9,78)$ & 0,98 (n.s.) \\
\hline $\begin{array}{l}\text { ESCALA DE } \\
\text { DEPRESIÓN (HRS) } \\
\text { (Rango 0-52) }\end{array}$ & $25,31(12,85)$ & $24,8(13,2)$ & $25,98(12,41)$ & 0,49 (n.s.) \\
\hline $\begin{array}{l}\text { ESCALA DE } \\
\text { AUTOESTIMA } \\
\text { (Rango 10-40) }\end{array}$ & $26,5(5,83)$ & $26,7(6,25)$ & $26,2(5,23)$ & 0,51 (n.s.) \\
\hline $\begin{array}{l}\text { INADAPTACIÓN } \\
\text { GLOBAL } \\
\text { (Rango } 0-6 \text { ) }\end{array}$ & $5,17(1,19)$ & $5,2(1,28)$ & $5,17(1,04)$ & 0,01 (n.s.) \\
\hline
\end{tabular}


da terapéutica. Tampoco en este caso hay diferencias significativas entre las dos submuestras.

El grado de ansiedad de las víctimas es elevado. En concreto, el $93 \%$ de la muestra supera la puntuación media de la población y el $86 \%$ supera el percentil 75 .

El nivel de sintomas depresivos presentado por las pacientes, tal como es recogido en el $B D I$ y en el HRS, es más bien alto. Nada menos que el $64 \%$ en el $B D I$ y el $68 \%$ en el HRS superan los puntos de corte establecidos. La gravedad de los sintomas es más clara en las manifestaciones conductuales y somáticas de la depresión (apatia, pérdida de interés, dificultades del sueño, retardo pșicomotor, etc.).

En lo que se refiere a la adaptación a la vida cotidiana, las víctimas de maltrato presentan un nivel muy alto de inadaptación. El maltrato interfiere claramente en el funcionamiento diario de estas personas. Por otra parte, las victimas muestran un grado de autoestima escaso, por debajo de la población normal.

\section{CONCLUSIONES}

En este trabajo se ha realizado un análisis descriptivo de una muestra amplia de mujeres maltratadas que han acudido a un programa de asistencia terapéutica. El perfil de la victima es una mujer casada, con una edad media de 38 años y perteneciente a una clase social media o baja. En cuanto a este último dato, los resultados obtenidos pueden reflejar un sesgo. Al haberse ofrecido un tratamiento gratuito en el marco de un Centro de Asistencia Psicológica para Víctimas de Violencia Familiar, cabe pensar que estén representadas mínimamente mujeres maltratadas de clases sociales más altas, que pueden utilizar otro tipo de recursos privados.

En lo que se refiere a las caracteristicas del maltrato, es destacable la larga duración del mismo, superior a 12 años por término medio. Se trata, por tanto, de un problema cronificado, que puede ser atribuido a diversas circunstancias: precariedad económica, dependencia emocional y baja autoestima personal, miedo a asumir el futuro en soledad, futuro incierto de los hijos, etc. Algunos sesgos cognitivos, como la vergüenza a relatar conductas degradantes o la creencia de que la violencia familiar atañe sólo al ámbito de lo privado, pueden asimismo retrasar la búsqueda de ayuda terapéutica.

El aislamiento social y la ocultación de lo ocurrido están presentes en casi el $60 \%$ de las victimas. Esta situación está favorecida por la presión del agresor y por la vergüenza social percibida por la victima, es decir, por el miedo al qué dirán. Sin embargo, el aislamiento genera una mayor dependencia del agresor, quien, a su vez, experimenta un aumento del dominio a medida que se percata del mayor aislamiento de la víctima.

No deja de ser significativo que el $36 \%$ de las victimas estén separadas o en trámites de separación. Quiere ello decir que el mero alejamiento de la situación de maltrato no soluciona los problemas surgidos como consecuencia de la violencia familiar.

Las victimas de maltrato psicológico, que viven la violencia en forma de críticas constantes y atentados a la autoestima, de amenazas de agresión, de chantaje con los hijos, de imposición de conductas degradantes o de atribución a las mujeres de la responsabilidad de las conductas violentas, constituyen casi la mitad de la muestra total $(43 \%)$. En ocasiones, el maltrato psicológico es el primer peldaño para la violencia física; en otras, sin embargo, es un tipo de violencia que se va a mantener a este nivel de forma crónica, sin traspasar este tipo de límites.

En el maltrato psicológico el comienzo suele ser más tardío, las relaciones sexuales forzadas son menos habituales, la extensión a los hijos de la violencia es menor y el número de denuncias es claramente inferior.

Hay algunos "mitos" destruidos en este estudio. Por ejemplo, se ha atribuido a las mujeres ciertos desequilibrios emocionales, como el ser masoquistas por naturaleza o el tener una necesidad de sufrir. Sin embargo, sólo un $9 \%$ de las mujeres estudiadas tenía una historia psiquiátrica anterior. Es más, las mujeres que han tenido una relación violenta tienden a no repetir este tipo de relaciones con otras futuras parejas.

Otra idea que no encuentra un apoyo empírico es la relacionada con la cadena de transmisión de la violencia. Sólo un $18 \%$ de las mujeres de este estudio han sufrido o sido testigos de maltrato en la familia de origen. 
Desde una perspectiva psicopatológica, el trastorno de estrés postraumático está presente en el $55 \%$ de la muestra ( $\sin$ distinciones entre ambos tipos de maltrato), que es un porcentaje similar al existente en las agresiones sexuales y más del doble del que se produce como consecuencia de cualquier otro tipo de delito, lo que resulta congruente con los datos obtenidos en otros estudios (cfr. Douglas, 1987; Rosewater, 1988; Walker, 1989, 1991; Houskamp y Foy, 1991; Dutton, 1992; Kusher, Riggs, Foa y Miller, 1992; DuttonDouglas, Burghardt, Perrin y Chrestman, 1994; Zubizarreta et al., 1994)

El trastorno de estrés postraumático se da con mayor intensidad cuando las víctimas están más inadaptadas, experimentan relaciones sexuales forzadas, sufren maltrato incluso durante los embarazos, no denuncian la situación en la que se encuentran y carecen de apoyo social y familiar.

Los sintomas de ansiedad y la depresión constituyen un cuadro clínico que está presente en más del $65 \%$ de la muestra, como ocurre también en otros estudios previos (Hilberman y Munson, 1977,1978; Walker, 1979; Jaffe, Wolfe, Telford y Austin, 1986; Straus, 1987; Rosewater, 1988; Campbell, 1989; McGrath, Keita, Strickland y Russo, 1990; Sato y Heiby, 1992; Campbell, Sullivan y Davidson, 1995). Lo que lleva a la depresión en estos casos es la pérdida de autoestima, el aislamiento social (con lo que implica de pérdida de actividades gratificantes), la indefensión aprendida -no se puede predecir o controlar la violencia, sobre todo cuando ésta es intermitente y entremezclada con periodos de arrepentimiento y ternura- y los sentimientos de culpa que pueden aflorar por las conductas que la mujer ha realizado para evitar o detener la violencia: mentir, encubrir al agresor, tener contactos sexuales a su pesar, consentir en el maltrato a los hijos, etc. La depresión puede quedar en algunos casos "amortiguada" por un nivel adecuado de autoestima (Cascardi y O'Leary, 1992).

El resultado de todos estos síntomas clinicos es una profunda inadaptación a la vida cotidiana y una interferencia grave en el funcionamiento cotidiano.

En resumen, la experiencia de violencia en el hogar, que además tiende a cronificarse, es una variable de riesgo para el desarrollo de trastornos emocionales. En todas las variables psicopatológicas analizadas el nivel de riesgo es tan alto en el maltrato físico como en el psicológico porque las victimas tienden a percibir este último de forma tanto o más dolorosa que el físico (Echeburúa et al., 1996: Follingstad, Rutledge, Serg, House y Ploek, 1990).

Conviene despejar en futuras investigaciones algunas incógnitas, como las diferencias individuales de los síntomas psicopatológicos resultantes de la violencia en el hogar o las variables predictoras del trastorno de estrés postraumático. Sólo de este modo se podrá efectuar una prevención adecuada y diseñar unas estrategias terapéuticas depuradas.

\section{REFERENCIAS BIBLIOGRÁFICAS}

American Psychiatric Association (1987). Diagnostic and statistical manual of mental disorders (3rd. ed. rev.). Washington, DC. A.P.A.

American Psychiatric Association (1994). Diagnostic and statistical manual of mental disorder (4th. ed.). Washington, DC. Author.

Beck, A.T., Rush, A.J., Shaw, B.F. y Emery, G. (1979). Cognitive Therapy for De-pression. New York. Guilford Press (traducción, DDB, 1983).

Campbell, J.C. (1989). A test of two explanatory modelsof women's responses to battering. Nursing-Research, 38, 18-24.

Campbell, R., Sullivan, C.M. y Davidson, W.S. (1995). Women who use domestic violence shelters: Changes in depression over time. Psychology of Women Quarterly, 19, 237-255.

Cascardi, M. y O'Leary, K.D. (1992). Depressive symptomatology, self-esteem, and self-blame in battered women. Joumal of Family Violence, $7,4,249-259$.

Corral, P., Echeburúa, E., Sarasua, B. y Zubizarreta, I. (1992). Estrés postraumático en ex combatientes y victimas de agresiones sexuales: nuevas perspectivas terapéuticas. Boletín de Psicologia, 35, 7-24.

Douglas, M.A. (1987). The battered woman syndrome. En D.J. Sonkin (eds.). Domestic violence on trial: therapeutic and legal dimensions of family violence. New York. Springer.

Dutton, M.A. (1992). Assessment and treatment of posttraumatic stress disorder among battered women. En D.W. Foy (ed.). Treating PTSD. Cognitive-behavioral strategies. New York. Guilford Press. 
Dutton-Douglas, M.A., Burghardt, K.J., Perrin, S.G. y Chrestman, K.R. (1994). Battered women's cognitive schemata. Journal of Traumatic Stress, 7, 237-255.

Echeburúa, E. (1995). Evaluación y tratamiento de la fobia social. Barcelona. Martínez Roca.

Echeburúa, E. y Corral, P. (1988). Escala de inadaptación. Edición experimental. No publicado.

Echeburúa, E., Corral. P., Sarasua, B. y Zubizarreta, I. (1996). Tratamiento cognitivo-conductual del trastorno de estrés postraumático en víctimas de maltrato doméstico: un estudio piloto. Análisis y Modificación de Conducta, 22, 627-654.

Echeburúa, E., Corral, P., Sarasua, B., Zubizarreta, I., y Sauca, D. (1990). Malos tratos y agresiones sexuales: lo que la mujer debe saber $y$ puede hacer. Servicio de Publicaciones de Emakunde/Instituto Vasco de la Mujer, Vitoria.

Echeburúa, E., Corral, P., Sarasua, B., Zubizarreta, I. y Sauca, D. (1994). Escala de gravedad de sintomas del trastorno de estrés postraumático. En E. Echeburúa (ed.) (1994). Personalidades violentas. Madrid. Pirámide.

Echeburúa, E. y Fernández-Montalvo, J. (1997). Tratamiento cognitivo-conductual de hombres violentos en el hogar. Análisis y Modificación de Conducta (en prensa).

Follingstad, D.; Rutledge, L.; Serg, B.; House, E. y Ploek, D. (1990). The role of emotional abuse in physical abusive relationships. Journal of Family Violence, 5, 107-120.

Hamilton, M. (1960). A rating scale for depression. Journal of Neurology, Neurosurgery and Psychiatry, 23, 53-62.

Hilberman, E. y Munson, K. (1977-1978). Sixty battered women. Victimology: An Internacional Journal, 2, 460-470.

Houskamp, B.M. y Foy, D.W. (1991). The assessment of posttraumatic stress disorder in battered wome. Journal of Interpersonal Violence, 6, 367-375.

Jaffe, P; Wolfe, D.A.; Wilson, S; Zak, L. (1986). Emotional and physical health problems of battered women. Canadian Journal of Psychiatry, 31, 625-629.

Jaffe, P., Wolfe, D. A., Telford, A. y Austin, G. (1986). The impact of police charges in incidents of wife abuse. Journal of Family Violence, 1, 3749.

Kemp, A, Rawlings, E. y Green, B.L. (1991). Posttraumatic stress disorder (PTSD). In battered women: A shelter sample. Journal of Traumatic Stress. 4, 137-148.

Kilpatrick, D.G. (1992). Posttraumatic stress for the DSM-IV and ICD-10: The introduction of a new diagnosis. Disorder of extrem stress. Paper presented at the world conference of the International Society for Traumatic Stress Studies, Amsterdam.

Kusher, M.G., Riggs, D.S., Foa, E.B. y Miller, S.M. (1992). Perceived controllability and development of posttraumatic stress disorder in crime victims. Behaviour Research and Therapy, 31, 105-110.

McGrath, E.; Keita, G.P.; Strickland, B. y Russo, N.F. (1990). Women and depression: Research, risk factors, and treatment issues. Washing, DC: American Psychological Association.

O'Leary, K.D. y Arias, I. (1988). Prevalence, correlates, and development of spous abuse. En R.D. Peters y R.J. McMahon (eds.). Social learning in system approaches to marriage and the family. New York. Brunner/Mazel.

Rojas Marcos, L. (1995). Las semillas de la violencia. Espasa-Calpe. Madrid.

Rosenberg, M. (1965). Society and the adolescent self-image. Princenton University Press. Princeton.

Rosewater, L.B. (1988). Battered or schizophrenic? Psychological tests cant't tell. En K. Yllo y M. Bograd (eds.). Feminist perspectives on wife abuse. Guilford. Beverly Hills, New York.

Rounsaville, B.J. y Lifton, N. (1983). A therapy group for battered women, en $M$. Rosenbaum (ed.). Handbook of Short-Term Therapy Groups, McGraw-Hill, New York.

Sato, R.A. y Heiby, E.M. (1992). Correlates of depressive symptoms among battered women. Journal of Family Violence, 7, 229-245.

Saunders, D.G. (1994). Child custody decisions in families experiencing woman abuse. Social Work, 39, 51-59.

Silber, E. y Tippet, J.S. (1965). Self-esteem: Clinical assessment and measurement validation. Psychological Reports, 16, 1017-1071.

Spielberger, C.D., Gorsuch, R.L. y Lushene, R.E. (1970). Manual for the State-Trait Anxiety Inventory. Palo Alto, CA. Consulting Psychologists Press.

Stark, E., y Flitcraft, A.H. (1988). Women and children at risk: a feminist perspective on child abuse. International Journal of Health Services, 18, 97-118.

Stith, S., Williams, M. y Rosen, K. (eds.) (1992). Psicosociologia de la violencia en el hogar. Bilbao. Desclée de Brouwer (original, 1990).

Straus, M.A. (1987). The Conflict Tactics Scale: an evaluation and new data on validity, reliability, norms and scoring methods. Paper presented at the 1987 Meeting of the National Council of Family Relations.

Straus, M.A. y Gelles, R.J. (1990). How violent are american families? Estimates from the National 
Family Violence Resurvey and other studies. En M.A. Straus y R.J. Gelles (eds.). Physical Violence in American Families. New Brunswick, NJ. Transaction Publishers.

Trimpey, M.L. (1989). Self-esteem and anxiety: Key issues in an abused women's support group. Special Issue: Family violence. Issues in Mental Health Nursing, 10, 297-308.

Vázquez, C. y Sanz, J. (1991). Fiabilidad y validez factorial de la versión española del Inventario de Depresión de Beck. III Congreso de Evaluación Psicológica. Barcelona.

Villavicencio, P. (1993). Mujeres maltratadas: conceptualización y evaluación. Clínica y Salud, 4, 215-228.
Walker, L.E. (1979). Battered women. New York: Harper y Row Publishers.

Walker, L.E. (1989). Psychology and violence against women. American Psychologist, 44, 695-702.

Walker, L.E. (1991). Posttraumatic stress disorder in women: diagnosis and treatment of battered woman syndrome. Psychotherapy, 28, 21-29.

Wodarski, J. (1987). An examination of spouse abuse: Practice issues for the profession. Clinical Social Work Journal, 15, 173- 187.

Zubizarreta, I., Sarasua, B., Echeburúa, E., Corral, P., Sauca, D y Emparanza, I. (1994). Consecuencias psicológicas del maltrato doméstico. En E. Echeburúa (ed.). Personalidades violentas. Madrid. Pirámide.

\section{NOTA}

${ }^{1}$ Agradecimientos. Este estudio se ha financiado gracias a un convenio de investigación entre la Universidad del Pais Vasco, el Instituto Vasco de la Mujer, las Diputaciones de Vizcaya y Alava y el Ayuntamiento de Vitoria. 